\title{
Efficacy of intensity-modulated radiation therapy for optic nerve sheath meningioma
}

\author{
Hiroyuki Sasano $^{1}$ (1) $\cdot$ Keigo Shikishima $^{1} \cdot$ Manabu Aoki $^{2} \cdot$ Tsutomu Sakai $^{1} \cdot$ Yuki Tsutsumi $^{2} \cdot$ Tadashi Nakano $^{1}$
}

Received: 11 April 2019 / Revised: 28 June 2019 / Accepted: 17 July 2019 /Published online: 3 August 2019

(C) The Author(s) 2019

\begin{abstract}
Purpose The present study examined the efficacy and complications associated with intensity-modulated radiation therapy (IMRT) for optic nerve sheath meningioma (ONSM) in 15 cases and compared visual function before and after treatment.

Methods Consecutively diagnosed patients with ONSM treated with IMRT were evaluated from 2012 to 2017 . We categorized ONSM with three growth patterns (diffuse, fusiform, or globular). Visual acuity, visual fields, and optic disc findings were assessed before and after IMRT. Ocular and systemic complications were evaluated during and after treatment.

Results The 15 patients selected for analysis ranged in age from 33 to 77 years. Post-treatment observation periods were 8 to 57 months. After IMRT, tumor enlargement was not detected in any eyes, and tumor reduction was seen in 2 eyes. At final posttreatment follow-up, eyes with fusiform and globular growth maintained better visual acuity compared with pre-treatment, whereas 2 of 5 eyes with diffuse growth showed reduced vision. Five eyes with no apparent optic disc abnormality maintained better visual acuity compared with pre-treatment, whereas 8 of 10 eyes with disc edema and atrophy remained stable or showed reduced vision. Improvements were seen in all 5 eyes with optic discs negative for pre-treatment abnormalities. Final posttreatment visual field abnormalities improved in 11 eyes. All adverse events identified during IMRT improved rapidly during the treatment period.

Conclusion IMRT for the treatment of ONSM achieved improvement and preserved visual function. In particular, early treatment with IMRT before the appearance of optic disc abnormalities can be more effective for improving visual function.
\end{abstract}

Keywords Optic nerve sheath meningioma $\cdot$ Intensity-modulated radiation therapy $\cdot$ Post-treatment complications $\cdot$ Post-treatment follow-up

\section{Introduction}

Optic nerve sheath meningioma (ONSM) is a relatively rare tumor, accounting for $1-2 \%$ of all meningiomas $[1,2]$. Developing from the arachnoid cap cells of the optic nerve sheath, these benign and slowly progressive tumors cause

Electronic supplementary material The online version of this article (https://doi.org/10.1007/s00417-019-04424-w) contains supplementary material, which is available to authorized users.

Hiroyuki Sasano

hiroyuki.3108@gmail.com

1 Department of Ophthalmology, The Jikei University School of Medicine, 3-25-8 Nishi-Shimbashi, Minato-ku, Tokyo 105-8461, Japan

2 Department of Radiology, The Jikei University School of Medicine, Tokyo, Japan optic nerve insult through compression and vascular compromise, resulting in severe visual impairment that may lead to blindness [3]. Tumor may grow intracranially through the optic canal to involve the optic chiasm and the contralateral optic nerve. Hence, it is of paramount importance to stop or reverse the growth of the tumor. In the treatment of ONSM, preservation of vision following tumor resection is known to be difficult, because surgery precipitates complications that damage the pial vascular plexus nourishing the optic nerve in many cases, leaving $95 \%$ of patients with severe postoperative visual impairment [1]. Surgery is currently indicated when esthetic issues arise following loss of vision or when intracranial tumor extension impacts prognosis for survival. In recent years, stereotactic radiotherapy (SRT), in the broad sense of the term, has been reported as an effective alternative to surgery $[4,5]$.

Intensity-modulated radiation therapy (IMRT) uses a device such as a multileaf collimator to adjust spatial and temporal radiation intensity and irradiate a precise focal target 
from multiple directions, thereby achieving optimal dose distribution on a three-dimensional plane. An advanced version of conventional stereotactic radiation therapy that is more effective and non-invasive, this new system reduces exposure to surrounding tissue by enabling modulation of dose intensity within the radiation field. Studies have already shown the advantages of IMRT for treating various intracranial tumors and skull base meningiomas [6-9]. The optic nerve is highly sensitive to radiation and therefore requires precision radiotherapy to avoid severe radiation-induced optic neuropathy. Detailed research into IMRT outcomes in the treatment of ONSM is lacking. As such, the present study examined the efficacy and complications associated with IMRT for ONSM by applying the technique to a large number of cases and comparing visual function before and after treatment.

\section{Patients and methods}

\section{Design}

This is single hospital-based, retrospective, observational study.

\section{Patients}

Analysis was performed of cases of ONSM that were between February 2012 and August 2017 in the Division of NeuroOphthalmology and Ocular Oncology Unit of the Department of Ophthalmology at the Jikei University Hospital. The observation period lasted until October 2017. Diagnosis of ONSM was based on (1) unilateral onset, (2) slowly progressive visual deterioration, (3) positive findings of a relative afferent pupillary defect, (4) optic disc findings, and (5) optic nerve swelling and tram-track sign detected on contrast-enhanced computed tomography (CT) and magnetic resonance imaging (MRI) of the orbit. Although the tramtrack sign can be also seen in sarcoidosis, perioptic neuritis, leptomeningeal carcinomatosis, lymphoma, leukemia, and orbital inflammation, these diseases exhibit rapid deterioration. We excluded subjects presenting acute onset or general complications. In this study, all patients had prolonged symptoms up to treatment. Although various morphological typologies have been proposed for tumor growth patterns, the present study categorized patterns into three types as diffuse (tubular), fusiform, or globular according to definitions used in several past studies $[1,3,10]$.

IMRT was indicated if patients exhibited at least one of the following clinical features: (1) reduced best-corrected visual acuity ( $\log$ MAR $>0$ ), (2) visual field abnormality, or (3) intracranial tumor extension. Eyes with visual acuity at or below the ability to count fingers at the initial examination were excluded.

\section{IMRT irradiation}

IMRT was performed in the Division of Radiation Therapy at the Jikei University Hospital using a Clinac linear accelerator (Varian Medical Systems, Palo Alto, CA) (Supplement 1). After patients were fixed by immobilization equipment (ShellTM), gross tumor volume (GTV) was delineated using CT and MRI fusion images. GTV was the gross demonstrable extent and location of ONSM. Clinical target volume (CTV) was identical to the GTV. Planning target volume (PTV) was defined as the CTV with a margin of $3 \mathrm{~mm}$. A 2-mm set-up margin was added to the organs at risk, such as the optic nerve, optic chiasm, retina, and pituitary gland. Cone beam CT (CBCT) was performed before each treatment using a 360degree rotation of the linear accelerator, and CBCT-based GTV was precisely adjusted to planning GTV. Irradiation was provided as 50.4-54.0 Gy in 28-30 fractions, the known tolerance dose for the optic nerve.

\section{Evaluation of treatment efficacy}

Visual acuity, visual fields, optic disc, and radiology findings were assessed before and after IMRT. Counting fingers and hand motions were quantified as a $\log$ MAR value of -2 . Improvement and deterioration of vision was defined as changes in $\log$ MAR values of $<-0.2$, and $>+0.2$, respectively, whereas stable vision was defined as $-0.2 \leq \log \mathrm{MAR} \leq+$ 0.2 . Visual fields were assessed using Goldmann perimetry performed by an expert examiner with extensive experience. Poor visual acuity due to central scotoma was difficult to evaluate with Humphry field analyzer. In addition, the evaluation of peripheral visual field defect was required. Therefore, we selected Goldmann perimetry first.

\section{Statistical analysis}

Using SPSS Statistics version 25 software (IBM, Tokyo, Japan), pre- and post-IMRT measurements of visual acuity were compared using the Wilcoxon signed-rank sum test.

\section{Results}

\section{Patients}

The 15 patients selected for analysis comprised 14 women and only 1 man, with ages ranging from 33 to 77 years (median, 49 years). Eight right eyes and 7 left eyes were affected, and disease duration from subjective symptoms to treatment ranged from 5 months to 25 years. Minimum and maximum post-treatment observation periods were 8 months, and 4 years and 9 months, respectively (median, 1 year and 11 months). Individual patient data are shown in Table 1. 
Table 1 Clinical data of all patients

\begin{tabular}{|c|c|c|c|c|c|c|c|c|}
\hline Case no. & Sex & $\begin{array}{l}\text { Affected } \\
\text { eye }\end{array}$ & $\begin{array}{l}\text { Age at start } \\
\text { of treatment }\end{array}$ & Symptoms & $\begin{array}{l}\text { Disease duration up } \\
\text { to start of treatment }\end{array}$ & $\begin{array}{l}\text { Tumor growth } \\
\text { pattern }\end{array}$ & Tumor and extension sites & IMRT dose \\
\hline 1 & $\mathrm{~F}$ & $\mathrm{R}$ & $52 \mathrm{Y}$ & Reduced visual acuity & $10 \mathrm{Y}$ & Fusiform & Anterior predominant & $51.0 \mathrm{~Gy} / 30 \mathrm{Fr}$ \\
\hline 2 & $\mathrm{~F}$ & $\mathrm{~L}$ & $46 \mathrm{Y}$ & Discomfort, proptosis & $5 \mathrm{M}$ & Fusiform & Posterior predominant & $52.2 \mathrm{~Gy} / 29 \mathrm{Fr}$ \\
\hline 3 & $\mathrm{~F}$ & $\mathrm{R}$ & $39 \mathrm{Y}$ & Pain with eye movement & $13 \mathrm{M}$ & Diffuse & & $50.4 \mathrm{~Gy} / 28 \mathrm{Fr}$ \\
\hline 4 & $\mathrm{~F}$ & $\mathrm{~L}$ & $42 \mathrm{Y}$ & Reduced visual acuity & $11 \mathrm{M}$ & Globular & Posterior predominant & $50.4 \mathrm{~Gy} / 28 \mathrm{Fr}$ \\
\hline 5 & $\mathrm{~F}$ & $\mathrm{R}$ & $41 \mathrm{Y}$ & Reduced visual acuity & $3 \mathrm{Y}$ & Diffuse & $\begin{array}{l}\text { Intracranial extension } \\
\text { (parasellar region) }\end{array}$ & $51.0 \mathrm{~Gy} / 30 \mathrm{Fr}$ \\
\hline 6 & $\mathrm{~F}$ & $\mathrm{R}$ & $33 \mathrm{Y}$ & Visual field defect & $10 \mathrm{M}$ & Fusiform & Central predominant & $51.0 \mathrm{~Gy} / 29 \mathrm{Fr}$ \\
\hline 7 & $\mathrm{~F}$ & $\mathrm{R}$ & $73 \mathrm{Y}$ & Reduced visual acuity & $7 \mathrm{M}$ & Diffuse & & $51.0 \mathrm{~Gy} / 30 \mathrm{Fr}$ \\
\hline 8 & M & $\mathrm{L}$ & $72 \mathrm{Y}$ & Reduced visual acuity & $2 Y$ & Fusiform & Posterior predominant & $50.4 \mathrm{~Gy} / 28 \mathrm{Fr}$ \\
\hline 9 & $\mathrm{~F}$ & $\mathrm{~L}$ & $49 \mathrm{Y}$ & Reduced visual acuity & $4 \mathrm{Y}$ & Fusiform & & $51.0 \mathrm{~Gy} / 30 \mathrm{Fr}$ \\
\hline 10 & $\mathrm{~F}$ & $\mathrm{~L}$ & $77 \mathrm{Y}$ & $\begin{array}{l}\text { Reduced visual acuity, } \\
\text { proptosis }\end{array}$ & $10 \mathrm{Y}$ & Fusiform & & $51.0 \mathrm{~Gy} / 30 \mathrm{Fr}$ \\
\hline 11 & $\mathrm{~F}$ & $\mathrm{~L}$ & $59 \mathrm{Y}$ & Reduced visual acuity & $25 \mathrm{Y}$ & Fusiform & Anterior predominant & $51.0 \mathrm{~Gy} / 30 \mathrm{Fr}$ \\
\hline 12 & $\mathrm{~F}$ & $\mathrm{R}$ & $49 \mathrm{Y}$ & Reduced visual acuity & $11 \mathrm{M}$ & Diffuse & $\begin{array}{l}\text { Posterior predominant, } \\
\text { Intracranial extension }\end{array}$ & $51.0 \mathrm{~Gy} / 30 \mathrm{Fr}$ \\
\hline 13 & $\mathrm{~F}$ & $\mathrm{~L}$ & $71 \mathrm{Y}$ & Exotropia, proptosis & $2 \mathrm{Y}$ & Globular & Posterior predominant & $51.0 \mathrm{~Gy} / 30 \mathrm{Fr}$ \\
\hline 14 & $\mathrm{~F}$ & $\mathrm{R}$ & $56 \mathrm{Y}$ & Reduced visual acuity & $3 \mathrm{M}$ & Diffuse & & $51.0 \mathrm{~Gy} / 30 \mathrm{Fr}$ \\
\hline 15 & $\mathrm{~F}$ & $\mathrm{R}$ & $47 \mathrm{Y}$ & Proptosis & $10 \mathrm{Y}$ & Fusiform & Anterior predominant & $51.0 \mathrm{~Gy} / 30 \mathrm{Fr}$ \\
\hline
\end{tabular}

$F$ female, $M$ male, $R$ right, $L$ left, $Y$ years, $M$ months, $G y$ Gray, $F r$ fraction

\section{Radiology findings}

In terms of ONSM morphology, 5 eyes showed diffuse growth with 2 eyes showing intracranial extension, 8 eyes showed fusiform growth with 1 eye showing intracranial extension, and 2 eyes showed globular growth (Table 1, Figs. 1, 2, and 3). After IMRT, tumor enlargement was not detected in any eyes, and tumor reduction was seen in 2 eyes. Intracranial invasion was not found in cases with intraorbital ONSM after IMRT.

\section{Optic disc findings}

Before treatment, swelling, atrophy, and optociliary shunt vessels were observed in the optic discs of 7, 3, and 5 eyes, respectively, whereas no abnormalities were found in 5 eyes (Table 2). After treatment, optic disc atrophy was detected in 3 eyes and anterior ischemic optic neuropathy was detected in 1 eye. No changes in optic discs findings were observed in the remaining 11 eyes. In addition, no post-treatment changes were seen in the 5 eyes with optic discs negative for pretreatment abnormalities.

\section{Visual acuity}

Immediate post-treatment visual acuity (IPostVA), which reflects effects and complications immediately after 28 to 30 times radiation treatment, improved in 5 eyes, remained stable in 9 eyes, and deteriorated in 1 eye (Table 3). No significant difference between pre-treatment visual acuity (PreVA) and IPostVA was detected $(p=0.050)$. Final post-treatment visual acuity (FPostVA) improved in 7 eyes, remained stable in 4 eyes, and deteriorated in 4 eyes. No significant difference between PreVA and FPostVA was detected $(p=0.330)$.

Among diffuse tumor, IPostVA improved in 1 eye and remained stable in 4 eyes, whereas FPostVA improved in 3 eyes, and deteriorated in 2 eyes (Fig. 4a). Among fusiform tumor, IPostVA improved in 3 eyes, remained stable in 4 eyes, and deteriorated in 1 eye, whereas FPostVA improved in 3 eyes, remained stable in 3 eyes, and deteriorated in 2 eyes (Fig. 4b). Among globular tumor IPostVA improved and remained stable in 1 eye each, whereas FPostVA improved and remained stable in 1 eye each (Fig. 4c). At final posttreatment follow-up, eyes with fusiform and globular growth maintained better visual acuity compared with pre-treatment levels, whereas 2 of 5 eyes with diffuse growth showed reduced vision (40\%) (Fig. 4d).

Among the 5 eyes negative for optic disc abnormalities, improvement was seen in 4 eyes, whereas 1 eye remained stable (Fig. 5a). Among the 7 cases of optic disc swelling, IPostVA remained stable in 6 eyes and deteriorated in 1 eye, whereas FPostVA improved in 1 eye, remained stable in 3 eyes, and deteriorated in 3 eyes (Fig. 5b). Among the three cases of optic disc atrophy, IPostVA improved in 1 eye and remained stable in 2 eyes, whereas FPostVA improved, remained stable, and deteriorated in 1 eye each (Fig. 5c). Figure 5d compares mean PreVA, IPostVA, and FPostVA among the three optic disc patterns (no apparent abnormality, 


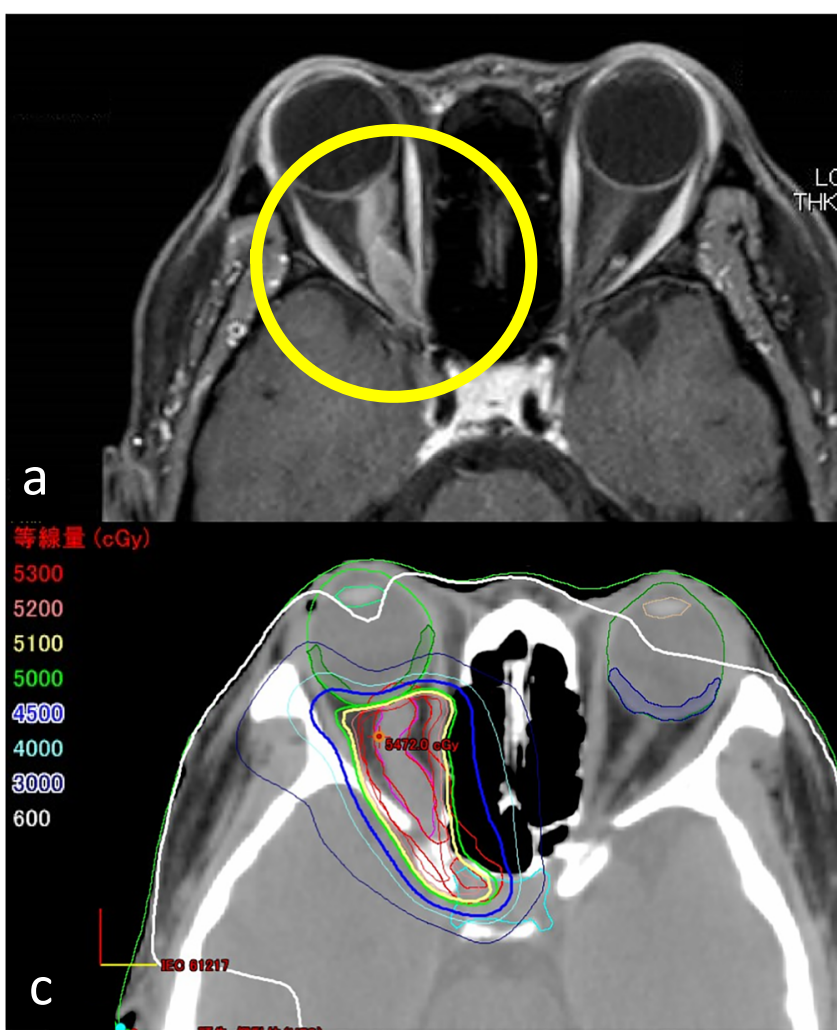

Fig. 1 Case 12 (diffuse type). a (Contrast-enhanced orbital MRI): finding of an intraorbital diffuse-type ONSM in the left eye with intracranial extension (circled area). b (Immediate post-IMRT GP of the right eye): inferior visual field loss (best-corrected logMAR visual acuity was 0.30 ).

edema, and atrophy). In all three patterns, vision improved immediately after treatment. At final post-treatment followup, all 5 eyes with no apparent abnormality maintained better visual acuity compared with pre-treatment levels, whereas 6 of 7 eyes with edema and 2 of 3 eyes with atrophy remained stable or showed reduced vision.

\section{Visual field}

Various visual field abnormalities were found to have accompanied ONSM (supplement 2). As for affected eyes, after treatment, abnormalities improved in 14 eyes. Improvements were seen in all 5 eyes with optic discs negative for pretreatment abnormalities. Final post-treatment visual field abnormalities improved in 11 eyes, deteriorated in 3 eyes, and were not assessed in 1 eye (Table 4). As for fellow eyes, scotomas were observed in 3 eyes before treatment, but improved after treatment.

\section{Adverse events}

Adverse events identified during IMRT included skin redness in 3 patients, lacrimation in 3 patients, eye/retrobulbar pain in 4 patients, heaviness in the rear of the eye in 2 patients,

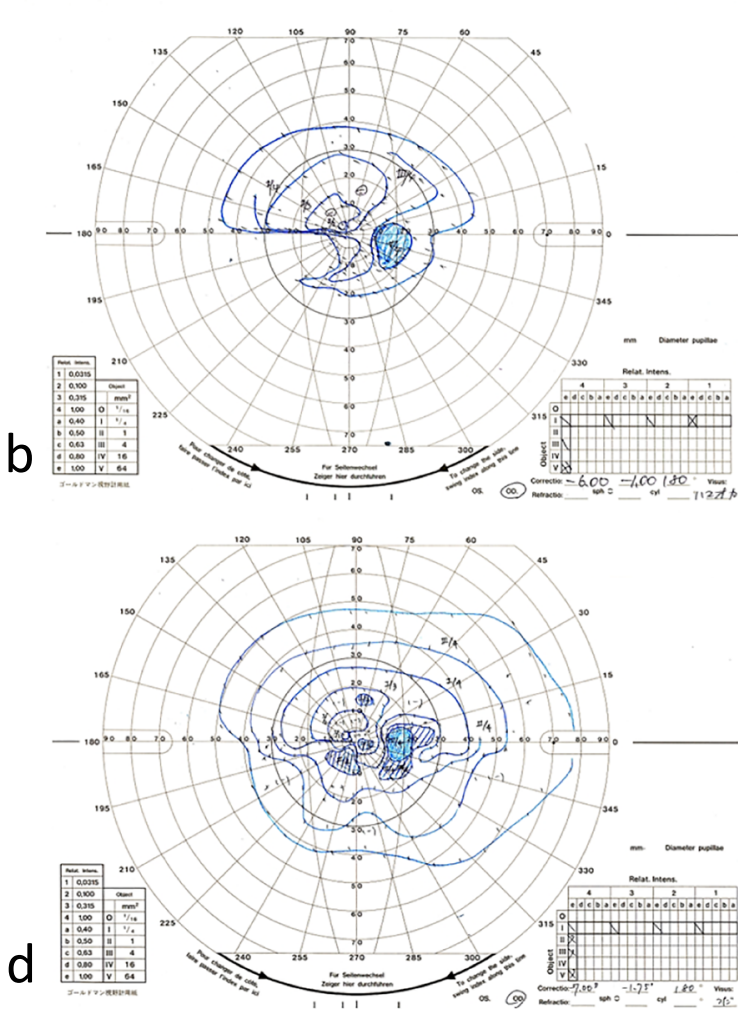

c (Orbital CT): IMRT dose distribution map. d (pre-IMRT GP of the right eye): inferior visual field improvement (best-corrected $\log$ MAR visual acuity was 0.15 )

headache in 4 patients, heavy-headedness in 1 patient, nausea in 2 patients, light-headedness in 2 patients, dizziness in 2 patients, fatigue/malaise in 11 patients, and dryness in the nose in 1 patient. All adverse events improved rapidly during the IMRT treatment period.

One eye (case 7) with a diffuse growth pattern that had maintained visual acuity immediately after IMRT developed an acute case of ischemic optic neuropathy on day 127 posttreatment. Another eye (case 1) showed reduced visual acuity due to a cataract on day 653 . No cases of systemic complications including endocrine disorders or hair loss were observed.

\section{Discussion}

In the context of visual function, increases in visual acuity from pre-treatment levels were achieved immediately after IMRT, and the absence of significant differences between PreVA and FPostVA indicates that vision was preserved in the overall sample. Furthermore, following IMRT, visual field improvements were seen in 14 eyes immediately after IMRT.

Although comparison of PreVA and IPostVA showed that IMRT was effective for all three tumor growth patterns, FPostVA of the entire sample was found to have decreased 


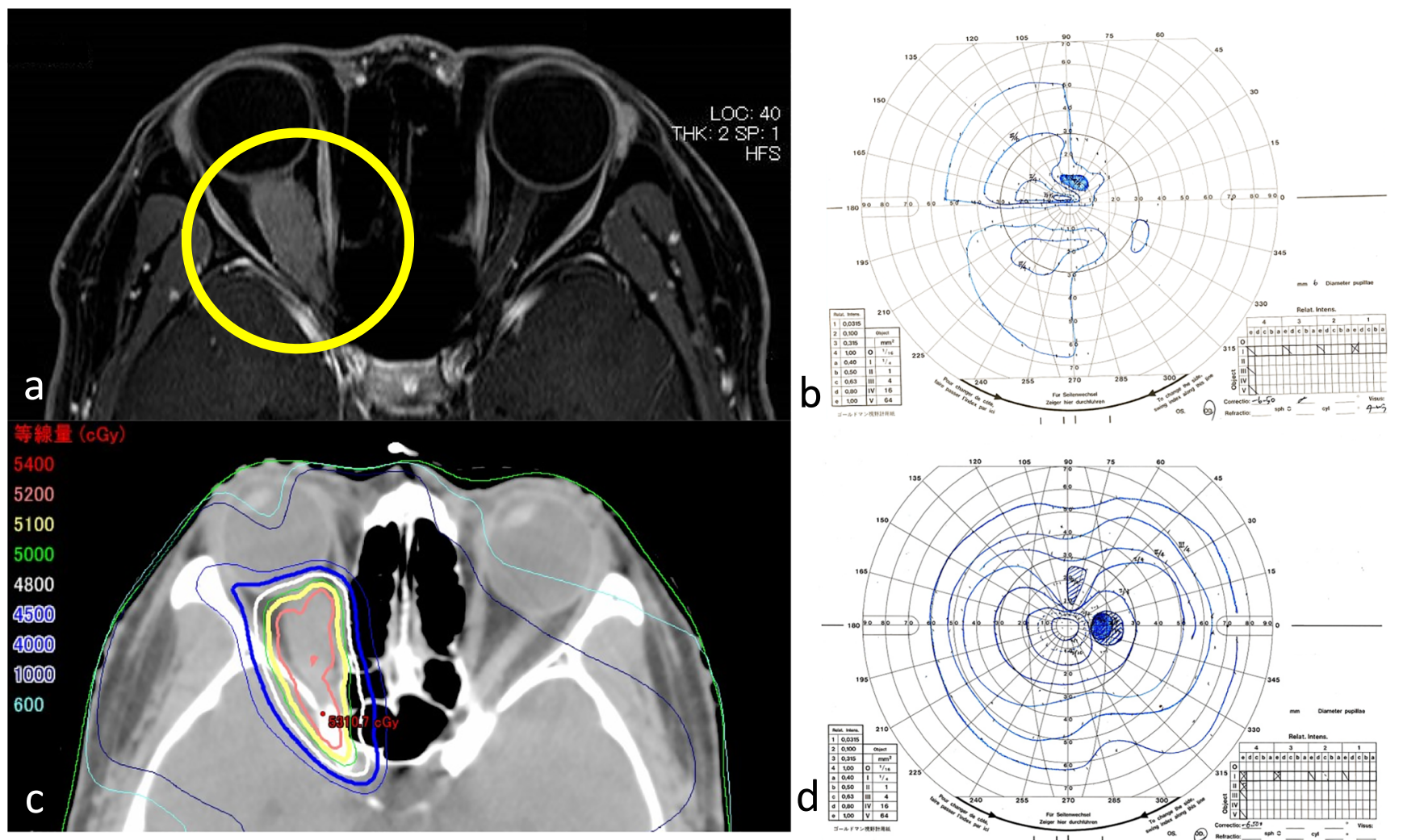

Fig. 2 Case 6 (fusiform type). a (Orbital MRI): finding of an intraorbital fusiform-type ONSM in the right eye (circled area). b (Pre-IMRT GP of the right eye): temporal visual field loss (best-corrected $\log M A R$ visual acuity was 0.40$)$. c (Orbital CT): IMRT dose distribution map. d (Immediate post-IMRT GP of the right eye): temporal visual field improvement (best-corrected $\log$ MAR visual acuity was -0.08 ) from IPostVA (Fig. 4a-c). FPostVA tended to decrease from PreVA among eyes with diffuse tumors, but it tended to increase among eyes with fusiform and globular tumors. ONSM is characterized by progressive visual loss, and although one study demonstrated that visual function prognosis is the worst in cases of diffuse tumor growth exhibiting apical expansion [3]. Other research into the prognoses of individual growth patterns is still lacking. The FPostVA findings of the present study suggest that IMRT is favorably indicated for fusiform and globular tumors. In contrast, it was difficult to reach any conclusions in terms of diffuse tumors, because the present sample was not compared with a control group undergoing natural disease progression. Therefore, the effectiveness of IMRT for treating diffuse tumors remains a priority moving forward. IMRT can be performed with high precision by adjusting to the shape of the lesion. Therefore, the difference in treatment effect among tumor growth pattern could result from the extent of the damage or preservation of the optic nerve depending on the shape of the tumors rather than the difference in the dose distribution.

With respect to optic disc findings, of the 5 eyes negative for pre-treatment optic disc abnormalities, there were significant improvements in IPostVA and field performance regardless of the degree of visual loss and visual field impairment present before IMRT; a decline in FPostVA compared with
IPostVA was observed in only 1 eye in the present study. On the other hand, post-treatment visual acuity and field performance for 10 eyes with pre-treatment optociliary shunt vessels and optic disc swelling and atrophy were inconsistent and ranged from improvement to no change to deterioration. Since eyes negative for optic disc abnormalities showed only a small reduction in FPostVA (i.e., late-stage vision), we believe that early treatment with IMRT before the appearance of atrophy, swelling, and other types of optic disc insult may lead to better outcomes for visual function.

In terms of SRT for ONSM, it has been reported that no relationship exists between the timing of radiotherapy and prognosis [10], that better visual acuity performance can be gained with early treatment [11], that treatment efficacy is greater in eyes with PreVA ranging from 20/40 to 20/30 [12], and that treatment is indicated when visual acuity is $\leq$ $20 / 40$ or when visual field impairment is detected [13].

Because the present study showed that treatment was effective regardless of the degree of visual loss, and FPostVA was better among eyes administered IMRT before developing optic disc impairment, we believe that early IMRT is desirable when patients experience subjectively reduced vision and visual field disturbances.

A search of the literature identified studies that examined the efficacy of IMRT for treating ONSM, and there were only 


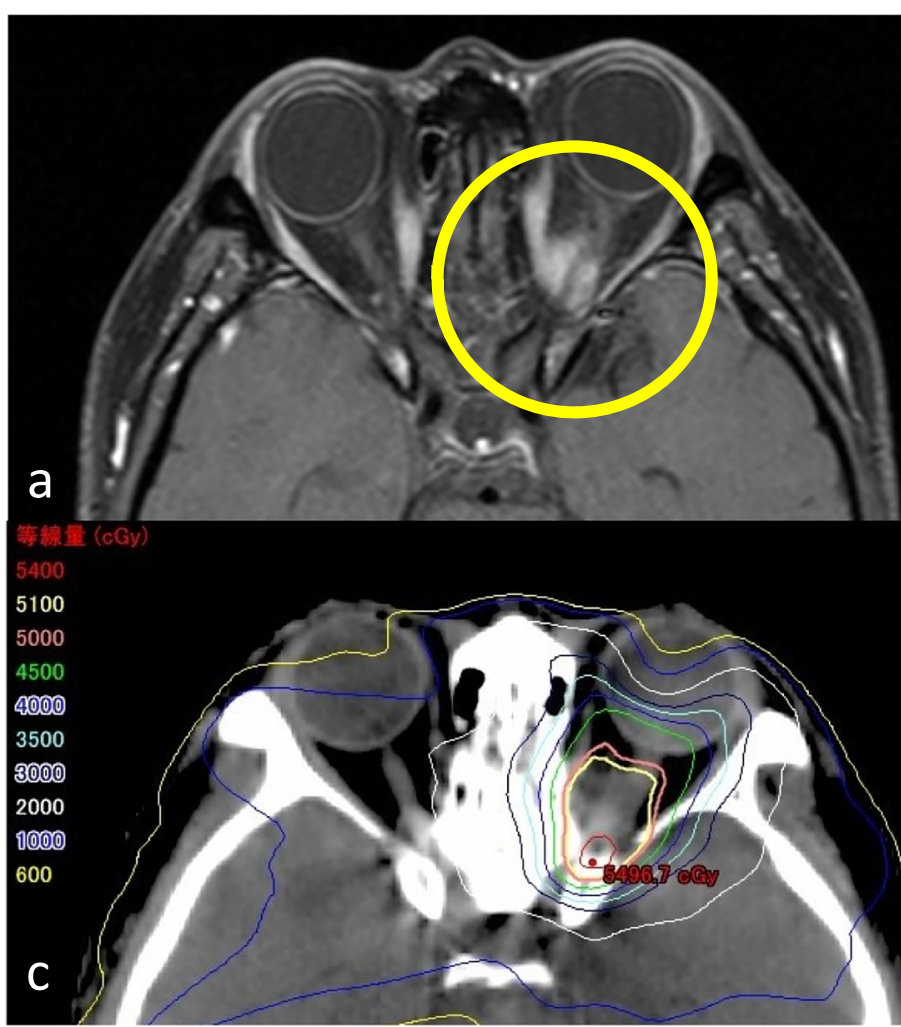

Fig. 3 Case 4 (globular type). a (Contrast-enhanced orbital MRI): finding of an intraorbital globular-type ONSM with the tram-track sign in the left eye (circled area). b (pre-IMRT GP of the left eye): finding of a central scotoma and a superior temporal scotoma (best-corrected logMAR visual

25 cases in total $[7,8,11,14-18]$. Furthermore, only 5 cases in total could be followed up for more than 5 years after IMRT as monotherapy $[16,18]$. According to these studies, IMRT

Table 2 Changes in optic disc findings from pre- to post-treatment

\begin{tabular}{clll}
\hline Case no. & $\begin{array}{l}\text { Optic disc finding } \\
\text { pre-treatment }\end{array}$ & OCSV & $\begin{array}{l}\text { Optic disc finding } \\
\text { post-treatment }\end{array}$ \\
\hline 1 & Swelling & + & Swelling \\
2 & Normal & - & Normal \\
3 & Swelling & + & Atrophy \\
4 & Normal & - & Normal \\
5 & Atrophy & - & Atrophy \\
6 & Normal & - & Normal \\
7 & Swelling & + & Atrophy, ION \\
8 & Atrophy & - & Atrophy \\
9 & Swelling & - & Atrophy \\
10 & Normal & - & Normal \\
11 & Swelling & + & Swelling \\
12 & Normal & - & Normal \\
13 & Atrophy & + & Atrophy \\
14 & Swelling & - & Atrophy \\
15 & Swelling & - & Swelling \\
\hline
\end{tabular}

$O C S V$ optociliary shunt vessel, $I O N$ ischemic optic neuropathy

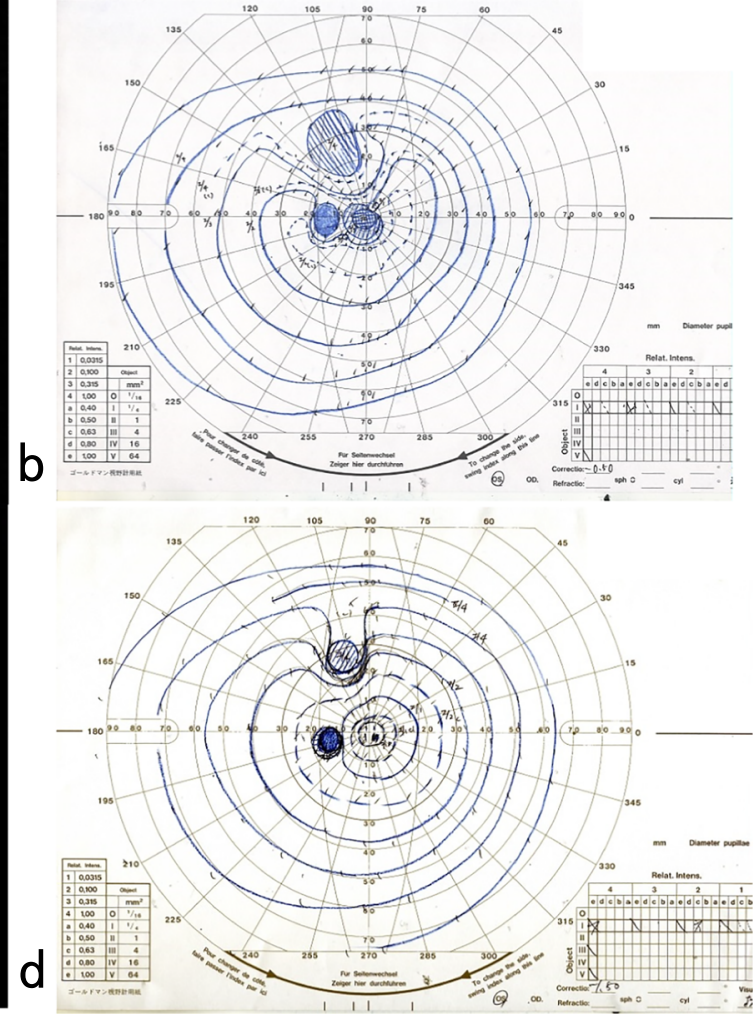

acuity was 0.52). c (Orbital CT): IMRT dose distribution map. d (Immediate post-IMRT GP of the left eye): disappearance of the central scotoma and reduction of the superior temporal scotoma (best-corrected $\log$ MAR visual acuity was -0.08 )

resulted in visual acuity improvement and stability in 19 eyes, no response in 3 eyes, and deterioration in 3 eyes, and it was associated with late adverse events, including lens opacification in 3 patients, dry eye in 3 patients, radiationinduced retinopathy in 2 patients with diabetes mellitus, keratitis in 2 patients, and blepharitis, otitis media with effusion, and early menopause in 1 patient each. The number of eyes examined in the present study is so far the greatest in a single institute.

Comparing different types of stereotactic irradiation techniques, SRT uses fractionated irradiation to deliver a uniform dose within the radiation field, IMRT delivers a non-uniform dose that mitigates exposure to proximal organs at risk, and stereotactic radiosurgery delivers a uniform dose within the radiation field in a single session. IMRT allows for more non-invasive treatment, because dose intensity can be modulated within the targeted field, thus lessening irradiation of surrounding tissue.

Various studies have reported the advantages of SRT for treating ONSM $[19,20]$, but they have also reported both ocular and systemic complications. IMRT is anticipated to achieve better local tumor control and visual improvement with a lower complication rate than conventional SRT [21, 22]. Acute complications observed in the present study disappeared soon after completion of IMRT. In terms of late 
Table 3 Changes in visual acuity from pre- to post-treatment

Case no. logMAR pre-treatment $\operatorname{logMAR}$ post-treatment Change (post-pre)

$\log$ MAR final Change (final-pre)

Final observation period

\begin{tabular}{|c|c|c|c|c|c|c|c|c|}
\hline 1 & 0.22 & 0.15 & -0.0669 & No change & 0.05 & -0.1761 & No change & $44 \mathrm{M}$ \\
\hline 2 & 0.30 & -0.08 & -0.3802 & Improved & 0.00 & -0.3010 & Improved & $46 \mathrm{M}$ \\
\hline 3 & 1.70 & 1.70 & 0.0000 & No change & 2.00 & 0.3010 & Deteriorated & $47 \mathrm{M}$ \\
\hline 4 & 0.52 & -0.08 & -0.6021 & Improved & 0.00 & -0.5229 & Improved & $52 \mathrm{M}$ \\
\hline 5 & 2.00 & 1.70 & -0.3010 & Improved & 1.70 & -0.3010 & Improved & $58 \mathrm{M}$ \\
\hline 6 & 0.40 & -0.08 & -0.4771 & Improved & -0.18 & -0.5740 & Improved & $27 \mathrm{M}$ \\
\hline 7 & 0.40 & 0.30 & -0.0969 & No change & 2.00 & 1.6021 & Deteriorated & $32 \mathrm{M}$ \\
\hline 8 & 0.30 & 0.22 & -0.0792 & No change & 0.52 & 0.2218 & Deteriorated & $24 \mathrm{M}$ \\
\hline 9 & 0.22 & 0.70 & 0.4771 & Deteriorated & 1.22 & 1.0000 & Deteriorated & $21 \mathrm{M}$ \\
\hline 10 & 0.52 & 0.30 & -0.2218 & Improved & 0.22 & -0.3010 & Improved & $21 \mathrm{M}$ \\
\hline 11 & 1.00 & 0.82 & -0.1761 & No change & 1.05 & 0.0458 & No change & $12 \mathrm{M}$ \\
\hline 12 & 0.30 & 0.15 & -0.1461 & No change & -0.08 & -0.3802 & Improved & $21 \mathrm{M}$ \\
\hline 13 & 0.05 & 0.05 & 0.0000 & No change & 0.15 & 0.1091 & No change & $11 \mathrm{M}$ \\
\hline 14 & 0.82 & 1.00 & 0.1761 & No change & 0.52 & -0.3010 & Improved & $13 \mathrm{M}$ \\
\hline 15 & 0.40 & 0.22 & -0.1761 & No change & 0.22 & -0.1761 & No change & $8 \mathrm{M}$ \\
\hline
\end{tabular}

$M$ months

*Hand motion (HM) and no light perception (NLP) are equivalent to $\log \mathrm{MAR}=2.00$
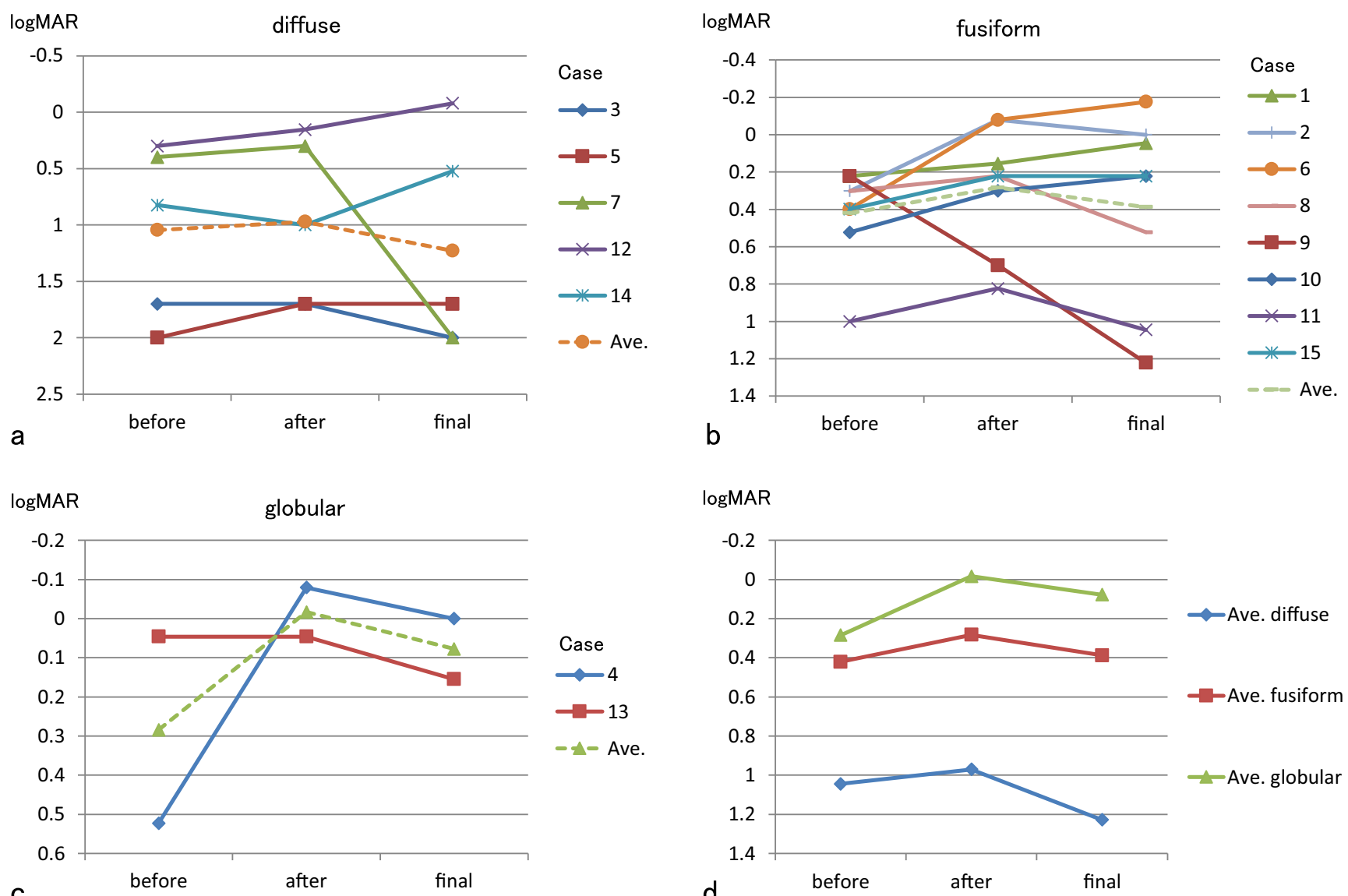

Fig. 4 Changes in pre-IMRT, immediate post-IMRT, and final post-IMRT visual acuity by tumor growth pattern. a Diffuse. b Fusiform. $\mathbf{c}$ Globular. d Comparison of means by tumor growth pattern 


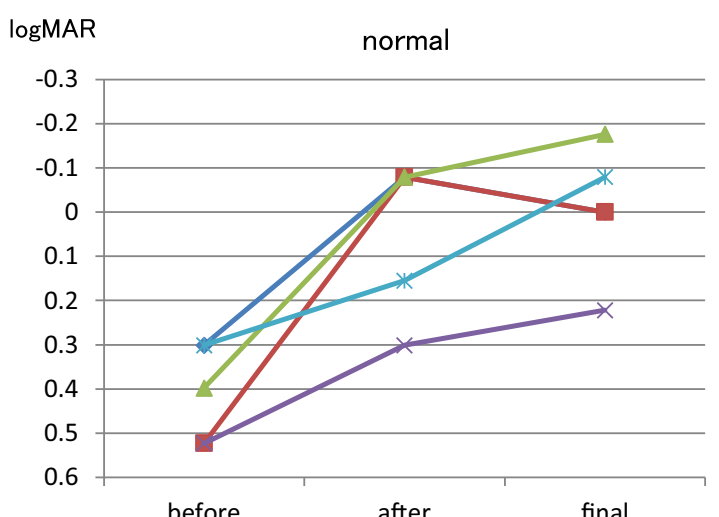

a

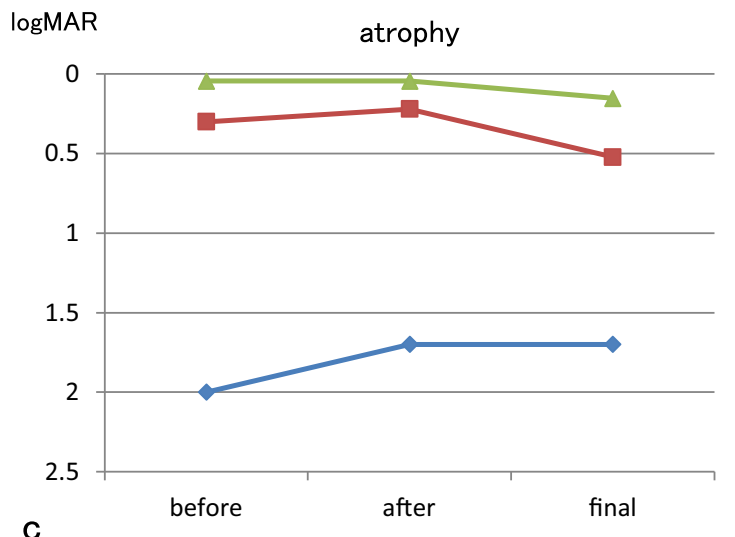

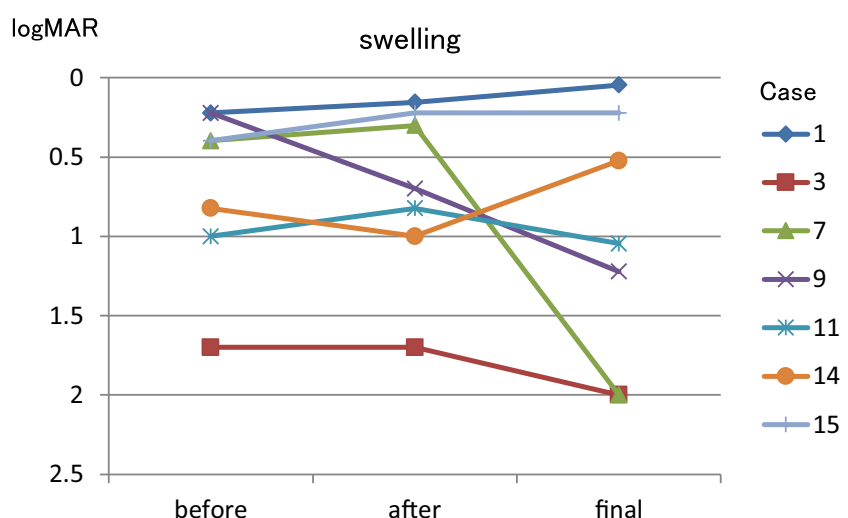

b

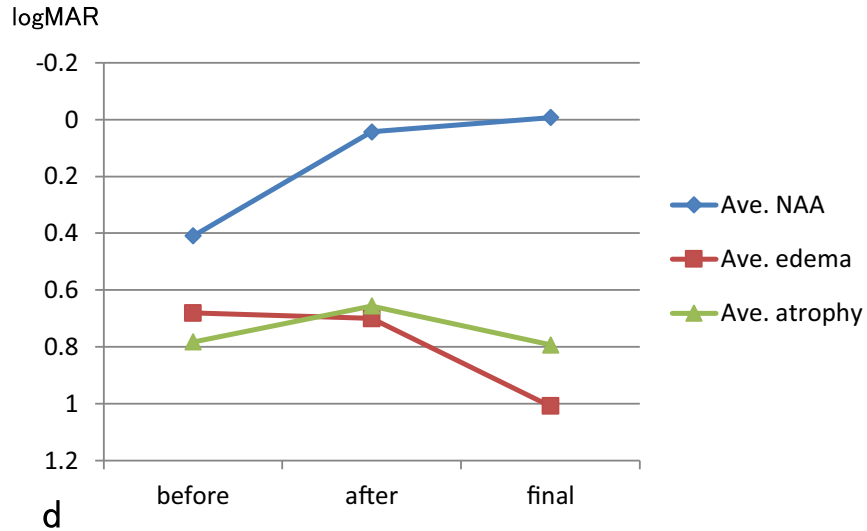

Fig. 5 Changes in pre-IMRT, immediate post-IMRT, and final post-IMRT visual acuity by optic disc findings. a No abnormality. b Swelling. c Atrophy. d Comparison of means by optic disc finding

Table 4 Changes of affected eye in visual field from pre- to post-treatment

\begin{tabular}{llll}
\hline Case no. & Visual field pre-treatment & Visual field post-treatment & Visual field final \\
\hline 1 & General reduction of sensitivity & Improved & Deteriorated (scotoma) \\
2 & Inferior visual field constriction & Improved & Improved \\
3 & Preservation of temporal and inferior temporal fields only & Improved (slight increase in sensitivity) & Deteriorated \\
4 & Central scotoma, superior scotoma & Improved (central scotoma disappearance, & Improved \\
5 & Preservation of superior field only & superior scotoma reduction) & Improved \\
6 & Temporal field loss, inferior field constriction & Improved & Improved \\
7 & Central scotoma, paracentral scotoma, inferior field constriction & Improved & Improved (central scotoma disappearance) \\
8 & Superior paracentral scotoma, nasal field constriction & Improved (of nasal field) & Deteriorated \\
9 & No data & Inferior field constriction & Improved \\
10 & Inferior paracentral scotoma & Improved (paracentral scotoma reduction) & Improved \\
11 & Preservation of temporal field only & Improved (remaining temporal field) & Improved \\
12 & Inferior field loss & Improved (of inferior field) & Improved \\
13 & Temporal field loss & Improved (temporal field expansion) & Improved \\
14 & Generalized visual field constriction & Improved & Improved \\
15 & Superior scotoma, nasal scotoma & Improved (nasal scotoma disappearance) & Improved \\
\hline
\end{tabular}


complications, 1 eye developed ischemic optic neuropathy after IMRT. Although hypopituitarism after SRT for ONSM is reported [21], there were no symptoms related to this complication in the present study.

We showed the efficacy of IMRT in a large number of cases in a single facility. However, several limitations of this study should be acknowledged. First, because of the various followup period, the time when the final visual function was evaluated is different in each case. Second, due to the short observation period, the late complications of IMRT have not been evaluated. The longest observation period is 4 years and 9 months in this study.

There are few reports of long-term prognosis of IMRT because IMRT is a novel radiation therapy compared with conventionally fractionated stereotactic radiotherapy and conformal radiotherapy. We will continue to follow up the cases and evaluate long-term post-treatment visual function and complications in further study.

IMRT for the treatment of ONSM achieved improvement and preservation of visual function. We believe that early treatment with IMRT before the appearance of optic disc abnormalities can be more effective for improving visual function, particularly among patients with fusiform and globular growth patterns. Moreover, the risk of serious post-treatment complications is considered low.

Funding This study was funded by the Japan Agency for Medical Research and Development (Grant Number JP17ck0106224).

\section{Compliance with ethical standards}

Conflict of interest Hiroyuki Sasano declares that he has no conflict of interest. Keigo Shikishima has received speaker honorariums from Santen Pharmaceutical Co., Ltd., Senju Pharmaceutical Co., Ltd., Johnson and Johnson K.K. and Cosmic Corp. Manabu Aoki declares that he has no conflict of interest. Tsutomu Sakai declares that he has no conflict of interest. Yuki Tsutsumi declares that she has no conflict of interest. Tadashi Nakano has received research grants from Crewt Medical Systems Inc., Kowa Pharmaceutical Co., Ltd., Tomey Corp, Senju Pharmaceutical Co., Ltd., Otsuka Pharmaceutical Co., Ltd., Merck Sharp and Dohme K.K., Pfizer Inc., Alcon Japan, Ltd., Santen Pharmaceutical Co., Ltd., Nidek Co., Ltd., Johnson and Johnson K.K. and Bayer Yakuhin, Ltd.

Ethical approval All procedures performed in studies involving human participants were in accordance with the ethical standards of the Jikei University School of Medicine Ethics Committee (No. 272488133) and with the 1964 Helsinki declaration and its later amendments or comparable ethical standards.

Informed consent Informed consent was obtained from all individual participants included in the study.

Open Access This article is distributed under the terms of the Creative Commons Attribution 4.0 International License (http:// creativecommons.org/licenses/by/4.0/), which permits unrestricted use, distribution, and reproduction in any medium, provided you give appropriate credit to the original author(s) and the source, provide a link to the Creative Commons license, and indicate if changes were made.

\section{References}

1. Dutton JJ (1992) Optic nerve sheath meningiomas. Surv Ophthalmol 37:167-183

2. Brain Tumor Registry of Japan 2005-2008 (2017) Neurol Med Chir 57:9-102. https://doi.org/10.2176/nmc.sup.2017-0001

3. Saeed P, Rootman J, Nugent RA, White VA, Mackenzie IR, Koornneef L (2003) Optic nerve sheath meningiomas. Ophthalmology 110:2019-2030. https://doi.org/10.1016/S01616420(03)00787-5

4. Adams G, Roos DE, Crompton JL (2013) Radiotherapy for optic nerve sheath meningioma: a case for earlier intervention? Clin Oncol 25:356-361. https://doi.org/10.1016/j.clon.2013.02.004

5. Brower JV, Amdur RJ, Kirwan J, Mendenhall WM, Friedman W (2013) Radiation therapy for optic nerve sheath meningioma. Pract Radiat Oncol 3:223-228. https://doi.org/10.1016/j.prro.2012.06. 010

6. Press RH, Prabhu RS, Appin CL, Brat DJ, Shu HK, Hadjipanayis C, Olson JJ, Oyesiku NM, Curran WJ, Crocker I (2014) Outcomes and patterns of failure for grade 2 meningioma treated with reduced-margin intensity modulated radiation therapy. Int $\mathrm{J}$ Radiat Oncol Biol Phys 88:1004-1010. https://doi.org/10.1016/j.ijrobp. 2013.12.037

7. Maclean J, Fersht N, Bremner F, Stacey C, Sivabalasingham S, Short S (2013) Meningioma causing visual impairment: outcomes and toxicity after intensity modulated radiation therapy. Int $\mathrm{J}$ Rradiat Oncol Biol Phys 85:e179-e186. https://doi.org/10.1016/j. ijrobp.2012.10.032

8. Grant W 3rd, Cain RB (1998) Intensity modulated conformal therapy for intracranial lesions. Med Dosim 23:237-241

9. Uy NW, Woo SY, Teh BS, Mai WY, Carpenter LS, Chiu JK, Lu HH, Gildenberg P, Trask T, Grant WH, Butler EB (2002) Intensitymodulated radiation therapy (IMRT) for meningioma. Int J Radiat Oncol Biol Phys 53:1265-1270

10. Saeed P, Blank L, Selva D, Wolbers JG, Nowak PJ, Geskus RB, Weis E, Mourits MP, Rootman J (2010) Primary radiotherapy in progressive optic nerve sheath meningiomas: a long-term follow-up study. Br J Ophthalmol 94:564-568. https://doi.org/10.1136/bjo. 2009.166793

11. Abouaf L, Girard N, Lefort T, D'Hombres A, Tilikete C, Vighetto A, Mornex F (2012) Standard-fractionated radiotherapy for optic nerve sheath meningioma: visual outcome is predicted by mean eye dose. Int J Radiat Oncol Biol Phys 82:1268-1277. https://doi.org/ 10.1016/j.ijrobp.2011.04.010

12. Landert M, Baumert BG, Bosch MM, Lutolf UM, Landau K (2005) The visual impact of fractionated stereotactic conformal radiotherapy on seven eyes with optic nerve sheath meningiomas. J Neuroophthalmol 25:86-91

13. Kennerdell JS, Maroon JC, Malton M, Warren FA (1988) The management of optic nerve sheath meningiomas. Am J Ophthalmol 106:450-457

14. Lee AG, Woo SY, Miller NR, Safran AB, Grant WH, Butler EB (1996) Improvement in visual function in an eye with a presumed optic nerve sheath meningioma after treatment with threedimensional conformal radiation therapy. J Neuroophthalmol 16: $247-251$

15. Smee RI, Schneider M, Williams JR (2009) Optic nerve sheath meningiomas-non-surgical treatment. Clin Oncol 21:8-13. https:// doi.org/10.1016/j.clon.2008.10.010

16. Lesser RL, Knisely JP, Wang SL, Yu JB, Kupersmith MJ (2010) Long-term response to fractionated radiotherapy of presumed optic nerve sheath meningioma. Br J Ophthalmol 94:559-563. https:// doi.org/10.1136/bjo.2009.167346

17. Inoue $\mathrm{T}$, Mimura $\mathrm{O}$, Masai $\mathrm{N}$, Ohashi $\mathrm{A}$, Ikenaga $\mathrm{K}$, Okuno $\mathrm{Y}$, Nishiguchi I, Oh R (2018) Early intervention using high-precision 
radiotherapy preserved visual function for five consecutive patients with optic nerve sheath meningioma. Int J Clin Oncol 23:826-834. https://doi.org/10.1007/s10147-018-1284-5

18. Jin J, Joo JD, Han JH, Yang HK, Hwang JM, Kim YJ, Kim IA, Kim CY (2018) Optic nerve sheath meningioma: preliminary analysis of the role of radiation therapy. Brain Tumor Res Treat 6:8-12. https:// doi.org/10.14791/btrt.2018.6.e2

19. Shapey J, Sabin HI, Danesh-Meyer HV, Kaye AH (2013) Diagnosis and management of optic nerve sheath meningiomas. J Clin Neurosci 20:1045-1056. https://doi.org/10.1016/j.jocn.2013.03. 008

20. Pacelli R, Cella L, Conson M, Tranfa F, Strianese D, Liuzzi R, Solla R, Farella A, Salvatore M, Bonavolonta G (2011) Fractionated stereotactic radiation therapy for orbital optic nerve sheath meningioma - a single institution experience and a short review of the literature. J Radiat Res 52:82-87

21. Paulsen F, Doerr S, Wilhelm H, Becker G, Bamberg M, Classen J (2012) Fractionated stereotactic radiotherapy in patients with optic nerve sheath meningioma. Int J Radiat Oncol Biol Phys 82:773778. https://doi.org/10.1016/j.jirobp.2010.11.018

22. Hamilton SN, Nichol A, Truong P, McKenzie M, Hsu F, Cheung A, Dolman P, Gete E, Ma R (2018) Visual outcomes and local control after fractionated stereotactic radiotherapy for optic nerve sheath meningioma. Ophthalmic Plast Reconstr Surg 34:217-221. https://doi.org/10.1097/IOP.0000000000000914

Publisher's note Springer Nature remains neutral with regard to jurisdictional claims in published maps and institutional affiliations. 